\title{
Effects of Polaron and Quantum Confinement on the Nonlinear Optical Properties in a GaAs/Ga $\mathbf{G}_{1-x} \mathbf{A l}_{x}$ As Quantum Well Wire
}

\author{
L. Caroline Sugirtham, ${ }^{1}$ A. John Peter, ${ }^{2}$ and Chang Woo Lee \\ ${ }^{1}$ Department of Physics, Fatima College, Madurai 625 018, India \\ ${ }^{2}$ Department of Physics, Government Arts College, Melur 625 106, Madurai, India \\ ${ }^{3}$ Department of Chemical Engineering, College of Engineering, Kyung Hee University, 1732 Deogyeong-daero, Giheung, Yongin, \\ Gyeonggi 446-701, Republic of Korea
}

Correspondence should be addressed to A. John Peter; a_johnpeter@rediffmail.com

Received 17 November 2013; Revised 26 January 2014; Accepted 10 February 2014; Published 11 March 2014

Academic Editor: Wen Lei

Copyright (C) 2014 L. Caroline Sugirtham et al. This is an open access article distributed under the Creative Commons Attribution License, which permits unrestricted use, distribution, and reproduction in any medium, provided the original work is properly cited.

\begin{abstract}
The binding energy of a polaron confined in a GaAs/ $\mathrm{Ga}_{1-x} \mathrm{Al}_{x}$ As quantum well wire is calculated within the framework of the variational technique and Lee-Low Pines approach. The polaron-induced photoionization cross section as a function of normalized photon energy for a on-centre donor impurity in the quantum wire is investigated. The oscillator strength with the geometrical effect is studied taking into account the polaron effects in a GaAs $/ \mathrm{Ga}_{0.8} \mathrm{Al}_{0.2}$ As quantum well wire. The effect of polaron on the third-order susceptibility of third harmonic generation is studied. Our theoretical results are shown to be in good agreement with previous investigations.
\end{abstract}

\section{Introduction}

The electronic properties and the potential applications for fabricating high performance novel optical devices in low dimensional semiconductor systems (LDSS) are quite interesting. The LDSSs can be produced with molecular beam epitaxy, metal organic chemical vapor deposition, chemical self-assemble technique, electron lithography, and molecular imprinted polymer. A lot of theoretical approaches have been devoted to study the nonlinear optical properties such as absorption coefficients, changes of refractive index, optical rectification coefficients, and second and third harmonic generation coefficients of these LDSSs [1-5]. These nonlinear optical properties pave the way for using any LDSS as probe for studying its electronic structure. The reduction in dimensionality in LDSS leads to a dramatic enhancement of optical nonlinearities. Electric field-induced polaron energy with a modified variational technique in a $\mathrm{GaAs} / \mathrm{Al}_{x} \mathrm{Ga}_{1-x} \mathrm{As}$ parabolic quantum well has been investigated by Zhao and Guo [6] who included the longitudinal optical phonons and the four branches of interface modes of phonon-polaritons in their study.
The study of phonon effect on any charged particle has been given due attention nowadays since the electronphonon interactions are considered to be more pronounced phenomena and expected to modify the electronic structure and transport properties of these systems since the phonons are confined in LDSSs. Especially, the electron-phonon interaction is enhanced by the spatial confinement and eventually one can expect the multiphonon processes. In particular, the electron-phonon interaction is necessary to comprehend the optical absorption spectra in materials. In fact, the effects of polaronic states are affected by the changes in the form developed by Fröhlich Hamiltonian describing the electronphonon interaction. Effects of electron and impurity ion LO phonon couples on the impurity states in cylindrical quantum wires have been dealt with [7]. Polaron effect in a wide band gap $\mathrm{GaN}$ material has been investigated by Zhang et al. [8], who studied the electron couples with both branches of longitudinal optical-like and transverse optical-like phonons in the presence of electric field.

Polaron-induced third-order nonlinear optical susceptibility in a GaAs quantum disk has been investigated [9]. 
Exciton-phonon scattering in quantum wires along with the geometrical confinement has been investigated earlier [10]. The ground state energy of an electron coupled with a Coulomb potential and a longitudinal-optical phonon field in a quantum wire has been studied [11]. Vasilevskiy et al. [12] proposed a nonperturbative and nonadiabatic approach for the calculation of the polaron effect on the Raman scattering which gives information about single particle excitations.

In the present work, the binding energy of a polaron confined in a GaAs $/ \mathrm{Ga}_{1-x} \mathrm{Al}_{x}$ As quantum well wire is calculated within the framework of the variational and Lee-Low Pines approach. The oscillator strength as a function of wire radius is studied with and without the phonon effects. The polaron-induced photoionization cross section as a function of normalized photon energy for on-centre donor impurity in the quantum wire is investigated. The effect of polarons on the third-order susceptibility of third harmonic generation is studied. The method followed is presented in Section 2 while the results and discussion are provided in Section 3.

\section{Model and Calculation}

Based on the effective mass approximation, the Hamiltonian of a hydrogenic shallow donor impurity located at the centre of a quantum well wire, $\mathrm{GaAs} / \mathrm{Ga}_{1-x} \mathrm{Al}_{x} \mathrm{As}$, is considered. An electron which interacts with the phonons described by the Fröhlich Hamiltonian is given by

$$
H=H_{e}+H_{\mathrm{ph}}+H_{e-\mathrm{ph}},
$$

where $H_{e}$ is the Hamiltonian of the electron without including phonons given by

$$
\begin{aligned}
H_{e}= & -\frac{\hbar^{2}}{2 m_{e}^{*}}\left[\frac{\partial^{2}}{\partial \rho^{2}}+\frac{1}{\rho} \frac{\partial}{\partial \rho}+\frac{\partial^{2}}{\partial z^{2}}+\frac{1}{\rho^{2}} \frac{\partial^{2}}{\partial \phi^{2}}\right] \\
& -\frac{e^{2}}{\varepsilon_{\infty} \sqrt{\rho^{2}+z^{2}}}+V(\rho),
\end{aligned}
$$

where $\varepsilon_{\infty}$ is the optical dielectric constant of the wire material and $m_{e}^{*}$ is the effective mass of electron defined as

$$
\frac{1}{m_{e}^{*}}=\frac{1}{m_{0}}+\frac{2 P^{2}}{3 \hbar^{2}}\left[\frac{2}{E_{g}}+\frac{1}{E_{g}+\Delta}\right]
$$

where $E_{g}$ is the band gap and $\Delta$ is the spin orbit energy of GaAs material. The confining potential, $V(\rho)$, is given as

$$
V(\rho)= \begin{cases}0, & 0 \leq \rho \leq R \\ V_{0}, & \rho>R\end{cases}
$$

where $V(\rho)$ is the barrier height corresponding to $60 \%$ of the difference between the forbidden band of inner and outer material of the wire with $\Delta E_{g}=\left(1.36 x+0.22 x^{2}\right) \mathrm{eV}$ [13]. Here, the optical dielectric constant describes the electron cloud screening:

$$
H_{\mathrm{ph}}=\sum_{\vec{q}} \hbar \omega_{\mathrm{LO}} a_{\vec{q}}^{+} a_{\vec{q}}
$$

where $a_{\vec{q}}^{+} a_{\vec{q}}$ and $b_{\vec{q}}^{+} b_{\vec{q}}$ are the phonon creation and annihilation operators with wave vector $\vec{q}$ with the frequency $\omega_{\mathrm{LO}}$. It describes noninteracting optical phonon system. These operators satisfy the commutation relations $\left[a_{\vec{q}}^{+} a_{\vec{q}}\right]=\delta_{q, q^{\prime}}$. This gives Bose statistics to the phonons. The energy value for $\mathrm{LO}$ phonon is taken as $\hbar \omega_{\mathrm{LO}}=36.75 \mathrm{meV}$ at $4.2 \mathrm{~K}$.

The electron-phonon interaction, $H_{e-\mathrm{ph}}$, is given by [14]

$$
H_{e-\mathrm{ph}}=\sum_{m l k_{z}}\left\{\left[V_{\mathrm{LO}}^{*}(r) e^{i m \phi} e^{i k_{z} z}-V_{\mathrm{LO}}^{*}\left(r_{i}\right)\right] a_{m l}^{+}\left(k_{z}\right)+\text { h.c }\right\} \text {, }
$$

where $V_{\mathrm{LO}}^{*}$ is the Fourier coefficient of the electron-phonon interaction, due to the LO phonons. The matrix elements, $V_{\mathrm{LO}}^{*}$, bring out the strength of the interaction and, in fact, depend on the wave vector. The coupling parameter, $V_{\mathrm{LO}}^{*}$, is given by

$$
V_{\mathrm{LO}}^{*}= \begin{cases}\Gamma_{\mathrm{LO}}^{* m l}\left(k_{z}\right) J_{m}\left(\frac{\chi_{m}^{l} r}{R}\right), & r \neq 0, \\ \Gamma_{\mathrm{LO}}^{* m l}\left(k_{z}\right), & r=0,\end{cases}
$$

where the coupling coefficient of the electron-phonon interaction is given by

$$
\Gamma_{\mathrm{LO}}^{* m l}\left(k_{z}\right)=\frac{i \sqrt{2} e\left(\hbar \omega_{\mathrm{LO}}\right)^{1 / 2}}{\sqrt{L}\left(\chi_{m}^{l^{2}}+k_{z}^{2} R^{2}\right)^{1 / 2} J_{m+1}\left(\chi_{m}^{l}\right)}\left(\frac{1}{\varepsilon_{0}}-\frac{1}{\varepsilon_{\infty}}\right)^{1 / 2},
$$

where $m=0, \pm 1, \pm 2, \ldots, l=1,2,3, \ldots$, where $L$ is the length of the quantum wire along the $z$-axis and $J_{m}$ is the $m$ th order Bessel function of the first order. $\chi_{m}^{l}$ is the $l$ th root of $J_{m}$.

We follow the variational method developed by Lee-Low Pines $[15,16]$ using the expression for unitary transformation as

$$
\begin{gathered}
U_{0}=\exp \left\{\left[\sum_{m, l, q_{z}}\left(a_{\vec{q}}^{+}+a_{\vec{q}}\right)\left[\frac{V_{\mathrm{LO}}(\rho)}{\hbar \omega_{\mathrm{LO}}}+\frac{V_{\mathrm{TO}}(\rho)}{\hbar \omega_{\mathrm{TO}}}\right]\right]\right\}, \\
U_{1}=\exp \left\{i\left[K_{z}-\sum_{m, l, q_{z}} q_{z} Z a_{\vec{q}}^{+} a_{\vec{q}}-\sum_{n, k_{z z}} q_{z} Z b_{\vec{q}}^{+} b_{\vec{q}}\right]\right\}, \\
U_{2}=\exp \left[\sum_{m, l, q_{z}} f_{m l}^{\mathrm{LO}}\left(q_{z}, z\right) a_{\vec{q}}^{+}-\text {h.c }\right] \\
+\exp \sum_{n, k_{z z}} f_{n}^{\mathrm{TO}}\left(q_{z}, z\right) b_{\vec{q}}^{+}-\text {h.c, }
\end{gathered}
$$

where $f_{m l}^{\mathrm{LO}}$ are the $f_{n}^{\mathrm{TO}}$ are the parameters to be determined by minimizing the expectation of the bound polaron Hamiltonian. Thus, the effective Hamiltonian for the bound polaron in the phonon vacuum state can be derived as

$$
H_{\text {eff }}=\left\langle 0\left|U_{2}^{-1} U_{1}^{-1} \widehat{H} U_{1} U_{2}\right| 0\right\rangle .
$$


The trial wave function for the taken system is the product of the wave function chosen as $\psi(r)$ and the phonon vacuum state $|0\rangle$. The trail wave function is chosen as

$$
\psi_{n l k}(\rho, \phi, z)= \begin{cases}N_{1} \exp (i k z) \exp ( \pm i l \phi) J_{l}\left(r_{n l} \rho\right), & \rho<R, \\ N_{1} \frac{J_{l}\left(r_{n l} R\right)}{k_{l}\left(b_{n l} R\right)} \exp ( \pm i l \phi) K_{l}\left(b_{n l} \rho\right), & \rho \geq R,\end{cases}
$$

where $N_{1}$ is the normalization constant and $r_{n l}$ and $b_{n l}$ are given by

$$
\begin{gathered}
r_{n l}=\sqrt{\frac{2 m_{e}^{*} E_{n l k}}{\hbar^{2}}}, \\
b_{n l}=\sqrt{\frac{2 m_{e}^{*}\left[V_{0}-E_{n l k}\right]}{\hbar^{2}}},
\end{gathered}
$$

where $E_{n l k}$ is the ground state lowest binding energy which is obtained as the first root of the transcendental equation.

The trial wave function, considering the Coulomb interaction, is given by

$$
\begin{aligned}
& \psi_{n l k}(\rho, \phi, z) \\
& =\left\{\begin{array}{r}
l N_{2} \exp (i k z) \exp ( \pm i l \phi) J_{l}\left(r_{n l} \rho\right) \exp \left(-\delta\left(\rho^{2}+z^{2}\right)^{1 / 2}\right), \\
\rho<R, \\
N_{2} \frac{J_{l}\left(r_{n l} R\right)}{k_{l}\left(b_{n l} R\right)} \exp ( \pm i l \phi) K_{l}\left(b_{n l} \rho\right) \exp \left(-\delta\left(\rho^{2}+z^{2}\right)^{1 / 2}\right), \\
\rho>R,
\end{array}\right.
\end{aligned}
$$

where $N_{2}$ is the normalization constant and $\alpha$ is the variation parameter.

The binding energy of the donor is defined as

$$
E_{b}=E_{0}-\langle H(\rho, \phi, z)\rangle_{\min },
$$

where $E_{0}$ is the free polaron energy and $\langle H(\rho, \phi, z)\rangle$ is obtained by minimizing the Hamiltonian (1) with respect to the variational parameter, $\delta$. The Schrödinger equation is solved variationally by finding $\langle H(\rho, \phi, z)\rangle$ and the binding energy of the donor in a quantum well wire is given by the difference between the energy with and without the Coulomb interaction.

In general, the photoionization cross section, in any low dimensional semiconductor system, is described as the optical transition of an electron from the impurity ground state to any one of the final subband states. In fact, it requires an adequate energy for the transition from one level to another level. The distribution of wave function is explained by this process. The expression for the photoionization process in the standard dipole approximation is given by [17]

$$
\begin{aligned}
& \sigma(\hbar \omega) \\
& =\left(\frac{E_{\mathrm{eff}}}{E}\right)^{2} \frac{n}{\varepsilon} \frac{4 \pi^{2}}{3} \eta \hbar \omega \sum_{f}\left|\left\langle\psi_{i}|\vec{r}| \psi_{f}\right\rangle\right|^{2} \delta\left(E_{f}-E_{i}-\hbar \omega\right),
\end{aligned}
$$

where $E_{\mathrm{eff}} / E$ is the effective field ratio taken as unity for simplicity, $n$ is the refractive index of the semiconductor, $\varepsilon$ is the dielectric constant of GaAs, $\eta$ is the fine structure constant, and $\hbar \omega$ is the photon energy. The energy conserving delta function by the Lorentzian is given by

$$
\delta\left(E_{f}-E_{i}-\hbar \omega\right) \longrightarrow \frac{\Gamma}{\pi\left[\left(E_{f}-E_{i}-\hbar \omega\right)^{2}+\Gamma^{2}\right]},
$$

where $\Gamma$ is the line width of the hydrogenic impurity for which the value is taken as $1 \mathrm{meV}$. The final state may be taken as

$$
\psi_{f}=A_{4}\left(\frac{2}{L S}\right)^{2} \exp \left(i \vec{k}_{\perp} \cdot \vec{\rho}\right) \sin \left(k_{11} \rho\right), \quad \rho<R,
$$

where $L$ is the length of the wire, $S$ is the surface area, $A_{4}$ is the normalization constant, and

$$
\exp \left(i \vec{k}_{\perp} \cdot \vec{\rho}\right)=J_{0}\left(k_{\perp} \rho\right)+2 \sum_{m=1}^{\infty} i^{m} J_{m}\left(k_{\perp} \rho\right) \cos \left(m\left[\theta-\theta_{k}\right]\right)
$$

$J_{0}, J_{m}$ are Bessel functions, and $\theta_{k}$ is the direction of vector $\vec{k}_{\perp}$. For light polarized in the $z$-direction, the transition from the impurity ground state to the final state is obtained as

$$
\begin{aligned}
\left\langle\psi_{f}|\rho| \psi_{i}\right\rangle & \\
=\hbar^{2} \times( & \left(2 m^{*}\left(E_{i}-E_{f}\right)\right) \\
& \left.\times\left\{\left\langle\psi_{f}\left|\frac{\partial}{\partial \rho}\right| \psi_{i}\right\rangle_{\rho<R}+\left\langle\psi_{f}\left|\frac{\partial}{\partial \rho}\right| \psi_{i}\right\rangle_{\rho>R}\right\}\right)^{-1},
\end{aligned}
$$

where $\omega$ is the angular frequency of the incident photon energy and $E_{i}$ and $E_{f}$ are the confined energies of initial and final states.

For any electronic system transitions, these calculations are important to compute the different linear and nonlinear optical properties. However, the dipole transitions are allowed using the selection rules $\Delta l= \pm 1$, where $l$ is the angular momentum quantum number. In addition to that the oscillator strength, which is related to the dipole transition, is expressed as

$$
P_{f i}=\frac{2 m^{*}}{\hbar^{2}} \Delta E_{f i}\left|M_{f i}\right|^{2}
$$

where $\Delta E_{f i}=E_{f}-E_{i}$ refers to the difference of the energy between the lower and upper states. $M_{f i}=2\left\langle\psi_{f}|\operatorname{er}| \psi_{i}\right\rangle$ is the electric dipole moment of the transition from $i$ state to $f$ state in the quantum wire. The matrix element is important for the calculation of different optical properties of the system related to the electronic transitions. The observation of oscillator strength is essential especially in the study of optical properties and they are related to the electronic dipole allowed absorptions. Moreover, the outcome of the results will be viewed on the fine structure of the optical absorption. 
The third-order nonlinear optical susceptibility $\chi^{(3)}$ which is related to the optical mixing between two incident light beams with different frequencies $\omega_{1}$ and $\omega_{2}$ is expressed as $[18,19]$

$$
\begin{aligned}
\chi^{3}\left(-2 \omega_{1}+\omega_{2}, \omega_{1},-\omega_{2}\right) & \\
= & \frac{-2 i \sigma_{s} e^{4}\left|\left\langle\psi_{i}|\vec{r}| \psi_{f}\right\rangle\right|^{4}}{\varepsilon_{0} \hbar^{3}\left[i\left(\omega_{0}-2 \omega_{1}+\omega_{2}\right)+\Gamma\right]\left[i\left(\omega_{2}-\omega_{1}\right)+\Gamma\right]} \\
& \times\left[\frac{1}{i\left(\omega_{0}-\omega_{1}\right)+\Gamma}+\frac{1}{i\left(\omega_{2}-\omega_{0}\right)+\Gamma}\right],
\end{aligned}
$$

where $\sigma_{s}$ is the density of electrons in the quantum well wire, $\varepsilon_{0}$ is the vacuum permittivity, $\Gamma=1 / \tau$ is the relaxation rate for states 1 and 2 , and $\hbar \omega$ is the photon energy. We have taken the relaxation rate as $1 \mathrm{ps}$ and the electron density is taken as $1 \times 10^{24} \mathrm{~m}^{-3}$. The above equation gives the expression for the third-order susceptibility of third-order harmonic generation which has been done by the compact-densitymatrix approach.

\section{Results and Discussion}

All material parameters are considered in atomic units in which $\hbar$ and $e^{2}$ are taken as unity. With the introduction of Rydberg scale, the effective Rydberg energy is defined as $R^{*}=m_{e}^{*} e^{4} / 2 \hbar^{2} \varepsilon_{0}^{2}$ and the effective Bohr radius as $R_{B}^{*}=$ $\hbar^{2} \varepsilon_{0} / m_{e}^{*} e^{2}$, and hence, the values of these quantities, for GaAs semiconductor, become $R^{*}=5.28 \mathrm{meV} R_{B}^{*}=103 \AA$. The parameters used in our calculations are $m_{e}^{*}=0.067 m_{0}$, where $m_{0}$ is the free electron mass, $\hbar \omega_{\mathrm{LO}}=36.75 \mathrm{meV}, \varepsilon_{0}=13.16$, $\varepsilon_{\infty}=10.89$, and the barrier height, $V_{0}$, is taken as $168.8 \mathrm{meV}$. The effective field ratio, $E_{\text {eff }} / E$, is assumed to be unity since the wave function is extended far from the impurity.

Figure 1 shows the donor binding energy of an impurity as a function of wire radius in a GaAs $/ \mathrm{Ga}_{0.8} \mathrm{Al}_{0.2}$ As quantum well wire with and without the polaron effects. Curve (1) represents with LO phonon contribution and curve (2) contributes to the phonon corrections. And the insert figure shows the corresponding polaronic shift due to LO phonons. It is observed that the donor binding energy monotonically increases with decreasing wire radius due to the geometrical confinement. When the wire radius decreases the electron wave function is more compressed in the quantum wire leading to an enhancement of binding energy. It is also observed that the coupling between the electron and phonon enhances the donor binding energy and this enhancement of binding energy is more pronounced for smaller wire radius. Thus, the ground state polaron energies increase with decreasing wire radius rapidly for narrow wires but slowly for larger wire radius. As a result the total binding energy incorporating the contributions of electron interaction with the LO phonon also enhances. The results are in good agreement with other investigators [20, 21]. It is found out from the insert figure that both the contributions of LO phonon with the polaron energy increase when the wire radius is decreased and this effect is more pronounced when the wire radius is decreased.

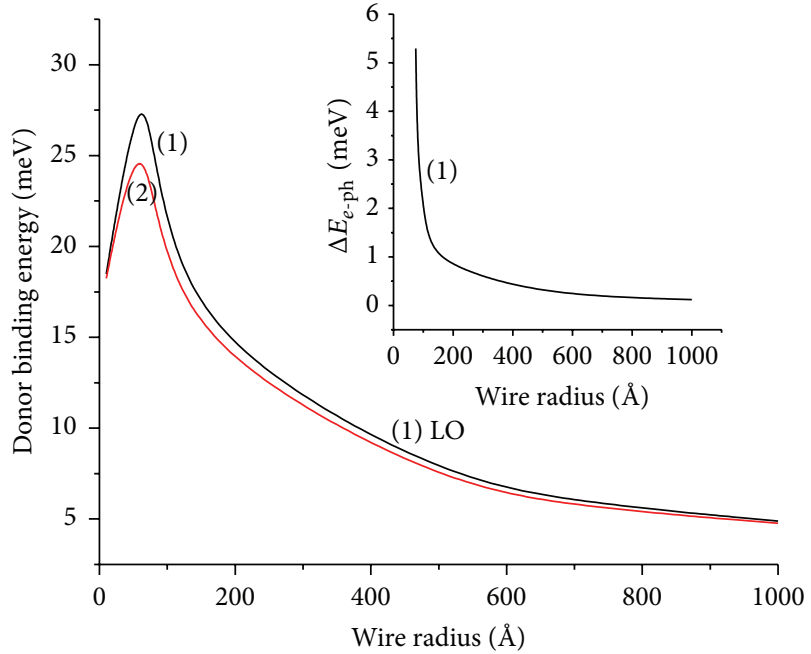

(1) With phonon corrections

(2) Without the phonon corrections

FIGURE 1: Binding energy of an impurity as a function of wire radius in a $\mathrm{GaAs} / \mathrm{Ga}_{0.8} \mathrm{Al}_{0.2}$ As quantum well wire with and without the polaron effects. Curve (1) represents for the LO phonon contribution and curve (2) contributes for the phonon corrections. The insert figure shows the corresponding polaronic shift due to LO phonons.

The phonon correction is found to have more influence for narrow wire in which the maximum binding energy is obtained. This is due to the fact that as the radius of the wire is reduced, the electron wave function becomes more localized and eventually the electron-phonon interaction is enhanced. Hence, it is concluded that the phonon contribution should not be neglected for any low dimensional semiconductor system.

In Figure 2, we represent the variation of oscillator strength as a function of wire radius in a GaAs/ $\mathrm{Ga}_{0.8} \mathrm{Al}_{0.2} \mathrm{As}$ quantum well wire with and without the polaron effects. Curve (1) denotes with LO phonon contribution and curve (2) represents without the phonon. It is observed that the phonon induced oscillator strength of the hydrogenic impurity decreases with the decrease in wire radius and the oscillator strength increases when the effects of phonon contribution are included. This is because the hydrogenic binding energy increases when the phonon contribution is included as seen earlier. The enhancement of energy difference of two different electronic states, due to the small transition probability between initial and final states, is observed with the inclusion of effects of phonon. Also, the reduction of recombination rate of the hydrogenic donor and the transition energy are observed when the wire radius is increased and further, the decay time also decreases when the geometrical confinement is reduced. The electron is more confined when the wire radius is decreased due to the pronounce effects of geometrical confinement. Eventually, the recombination rate is reduced [22].

In Figure 3, we display the variation of photoionization cross section as a function of normalized photon energy with and without the polaron effect for a $50 \AA$ wire radius 


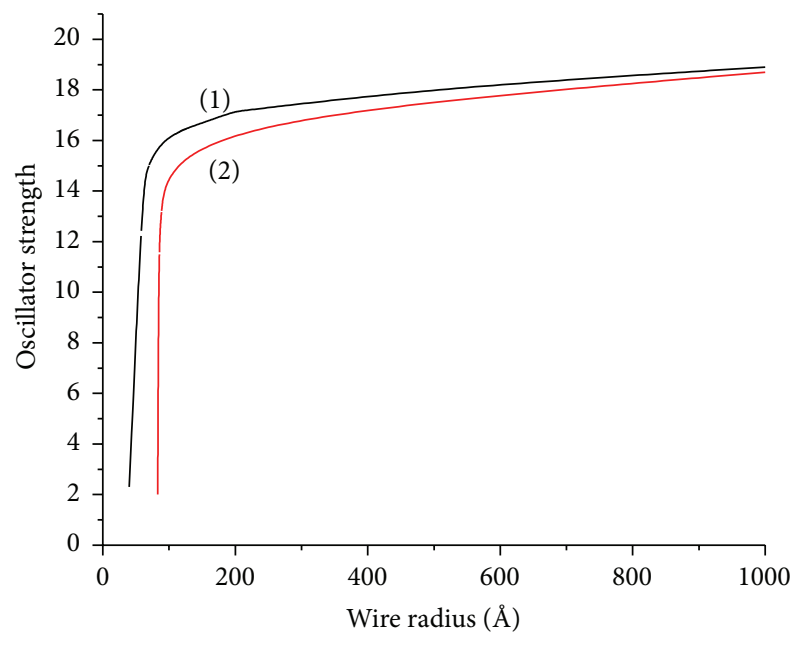

(1) With phonon contribution

(2) Without phonon contribution

FIGURE 2: Variation of oscillator strength as a function of wire radius in a $\mathrm{GaAs} / \mathrm{Ga}_{0.8} \mathrm{Al}_{0.2}$ As quantum well wire with and without the polaron effects. Curve (1) denotes with LO phonon contribution and curve (2) represents without the phonon contribution.

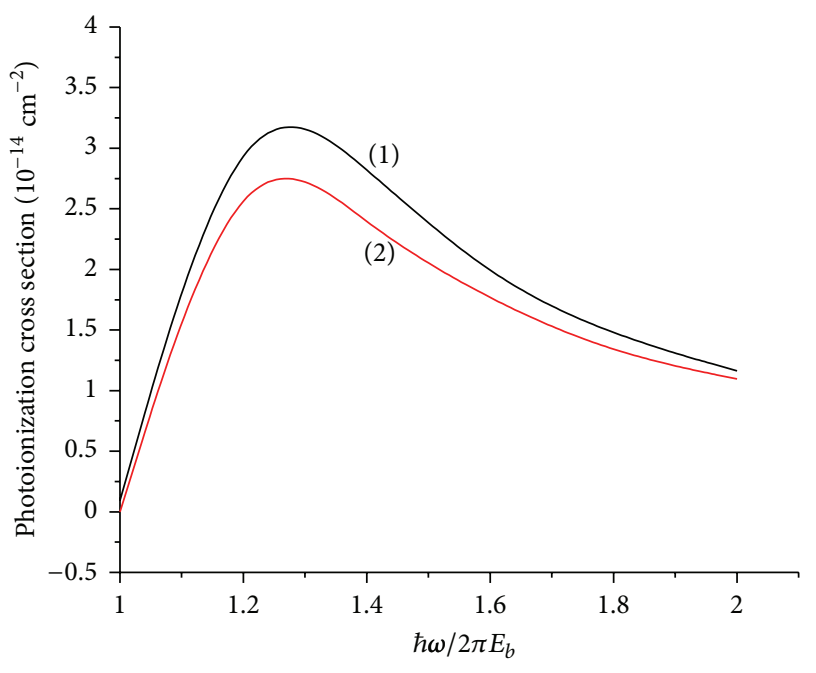

(1) Without the phonon contribution

(2) With phonon contribution

FIGURE 3: Variation of photoionization cross section as a function of normalized photon energy with and without the polaron effect for a $50 \AA$ wire radius in a $\mathrm{GaAs} / \mathrm{Ga}_{0.8} \mathrm{Al}_{0.2}$ As quantum well wire.

in a $\mathrm{GaAs} / \mathrm{Ga}_{0.8} \mathrm{Al}_{0.2}$ As quantum well wire. The photoionization cross section rises at lower energies whereas it decreases smoothly for larger photon energies [23]. It is because both the subband energies and the impurity binding energy increase with decreasing wire radius, the frequency at which the photoionization cross section peaks, increases with decreasing wire size. This figure clearly brings out the quantum size effect. Further, it is found that the magnitude of the photoionization cross section becomes higher and shifts

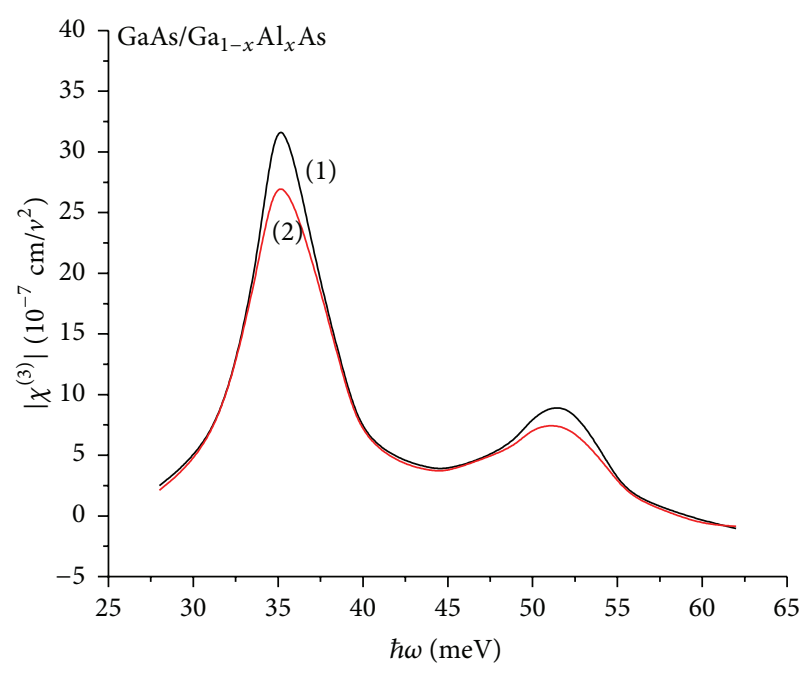

(1) Without phonon contribution

(2) With phonon contribution

FIGURE 4: Variation of third-order susceptibility of third harmonic generation as a function of photon energy with and without the polaron effects in a $\mathrm{GaAs} / \mathrm{Ga}_{0.8} \mathrm{Al}_{0.2}$ As quantum well wire.

towards the higher photon energies when the polaronic effect is included. It is due to the enhancement of optical matrix element between the initial and final states. It results in the increase of magnitude of the photoionization cross section.

Figure 4 shows the plot of variation of third-order susceptibility of third harmonic generation as a function of photon energy with and without considering the effects of electron-phonon interactions. In this figure, curve (1) refers to without the phonon contribution and curve (2) denotes LO phonon contribution. $50 \AA$ wire radius of a $\mathrm{GaAs} / \mathrm{Ga}_{0.8} \mathrm{Al}_{0.2}$ As quantum well wire is taken with $\hbar \omega_{0}=$ $50 \mathrm{meV}$. It is observed that the susceptibility has two peaks and the contribution of phonon makes the resonant peak towards higher region of energies [24, 25]. Further, it is noted that the magnitude of the resonant peak moves towards the higher energies with the increase of phonon effects. The coefficient of third-order susceptibility of third harmonic generation has been enhanced with the polaronic effects [25]. The relaxation time has more influence on the thirdorder susceptibility. It is found that the coefficient of thirdorder susceptibility with considering the polaron effect is 1.5 times larger than obtained without considering the electronphonon interaction. It is due to the enhancement of matrix element. LO phonons enhance third-order nonlinear optical susceptibilities of third harmonic generation. And hence, it is concluded that the contribution of phonon has more influence on the resonant peak of third-order susceptibility of third harmonic generation.

In conclusion, the polaron-induced binding energy has been computed in a $\mathrm{GaAs} / \mathrm{Ga}_{1-x} \mathrm{Al}_{x}$ As quantum well wire. Numerical calculations have been carried out using variational method within the single band effect mass approximation and Lee-Low Pines approach. The effects on oscillator strength with the spatial confinement have been dealt with. 
The polaron-induced photoionization cross section as a function of normalized photon energy for on-centre donor impurity in the quantum wire has been investigated. The polaron-induced third-order susceptibility of third harmonic generation has been investigated. The results show that the donor binding energy is enhanced by the electron-phonon interactions, the effect of phonon contribution is pronounced for smaller wire radii, and the coefficient of third-order susceptibility of third harmonic generation has been enhanced with the polaronic effects. The effects of phonon should be taken into account for narrow wires. We hope that the present work would stimulate further investigations on nonlinear optical properties in a weak polar material and the lattice dynamic properties in the near future.

\section{Conflict of Interests}

The authors declare that there is no conflict of interests regarding the publication of this paper.

\section{References}

[1] G. Rezaei, M. J. Karimi, and A. Keshavarz, "Excitonic effects on the nonlinear intersubband optical properties of a semiparabolic one-dimensional quantum dot," Physica E, vol. 43, no. 1, pp. 475-481, 2010.

[2] W. Xie, "Diploe-allowed optical absorption of an exciton in a spherical parabolic quantum dot," Optics Communications, vol. 282, no. 13, pp. 2604-2607, 2009.

[3] M. Şahin, “Third-order nonlinear optical properties of a oneand two-electron spherical quantum dot with and without a hydrogenic impurity," Journal of Applied Physics, vol. 106, Article ID 063710, 2009.

[4] I. Karabulut and S. Baskoutas, "Linear and nonlinear optical absorption coefficients and refractive index changes in spherical quantum dots: effects of impurities, electric field, size, and optical intensity," Journal of Applied Physics, vol. 103, no. 7, Article ID 073512, 2008.

[5] S. Winter, M. Zielinski, D. Chauvat, J. Zyss, and D. Oron, "The second order nonlinear susceptibility of quantum confined semiconductors-a single dot study," Journal of Physical Chemistry C, vol. 115, no. 11, pp. 4558-4563, 2011.

[6] F.-Q. Zhao and Z.-Z. Guo, "Electric field effects on polarons with spatially dependent mass in parabolic quantum wells," International Journal of Modern Physics B, vol. 18, no. 22, pp. 2991-2999, 2004.

[7] Z. Zhao and X. Liang, "Effects of electron-and impurity-ion-LO phonon couples on the impurity states in cylindrical quantum wires," Journal of Semiconductors, vol. 30, no. 6, Article ID 062002, 2009.

[8] B. Zhang, Z. Yan, and M. Zhang, "Bound polaron in a strained wurtzite $\mathrm{GaN} / \mathrm{Al}_{x} \mathrm{Ga}_{1-x} \mathrm{~N}$ cylindrical quantum dot," Journal of Semiconductors, vol. 32, no. 6, Article ID 062003, 2011.

[9] C.-H. Liu, K.-X. Gua, C.-Y. Chen, and B.-K. Ma, "Polaron effects on the third-order nonlinear optical susceptibility in a quantum disk," Physica E, vol. 15, no. 4, pp. 217-228, 2002.

[10] S.-D. Liang, C. Y. Chen, S. C. Jiang, and D. L. Lin, "Size effect on exciton-phonon scattering in quantum wires," Physical Review $B$, vol. 53, no. 23, pp. 15459-15461, 1996.
[11] Q. H. Chen, Z. B. Wang, F. L. Wu et al., "Variational pathintegral study on bound polarons in parabolic quantum dots and wires," Chinese Physics Letters, vol. 18, no. 5, p. 668, 2001.

[12] M. I. Vasilevskiy, R. P. Miranda, E. V. Anda, and S. S. Makler, "Polaron effect on Raman scattering in semiconductor quantum dots," Semiconductor Science and Technology, vol. 19, no. 4, pp. S312-S315, 2004.

[13] C. Bosio, J. L. Staehli, M. Guzzi, G. Burri, and R. A. Logan, "Direct-energy-gap dependence on Al concentration in $\mathrm{Al}_{x} \mathrm{Ga}_{1-x}$ As," Physical Review B, vol. 38, no. 5, pp. 3263-3268, 1988.

[14] P. M. Platzman, "Ground-state energy of bound polarons," Physical Review, vol. 125, no. 6, pp. 1961-1965, 1962.

[15] W. J. Huyberechts, "Internal excited stateof the optical polaron," Journal of Physics C, vol. 10, no. 19, p. 3761, 1977.

[16] T. Lu and Y. Zheng, "Polaron properties in quantum wells," Physical Review B, vol. 53, no. 3, pp. 1438-1445, 1996.

[17] M. Lax, "Photoconductivity," in Proceedings of the Atlantic City Conference on Photoconductivity, R. G. Breckenridge, B. R. Russell, and E. E. Hahn, Eds., p. 111, John Wiley \& Sons, New York, NY, USA, 1954.

[18] W. Xie, "Third-order nonlinear optical susceptibility of a donor in elliptical quantum dots," Superlattices and Microstructures, vol. 53, pp. 49-54, 2013.

[19] T. Takagahara, "Excitonic optical nonlinearity and exciton dynamics in semiconductor quantum dots," Physical Review B, vol. 36, no. 17, pp. 9293-9296, 1987.

[20] Z. Zengru and L. Xixia, "Effects of electron- and impurityion-LO phonon couples on the impurity states in cylindrical quantum wires," Journal of Semiconductors, vol. 30, no. 6, Article ID 062002, 2009.

[21] R.-Q. Wang, H.-J. Xie, K.-X. Guo, Y.-B. Yu, and Y.-Q. Deng, "Polaronic effects of an exciton in a cylindrical quantum wire," Communications in Theoretical Physics, vol. 43, no. 1, pp. 169174, 2005.

[22] J. H. You, J. T. Woo, D. U. Lee, T. W. Kim, K. H. Yoo, and H. L. Park, "Dependence of optical gain and interband transitions on the CdTe well width and temperature for CdTe/ZnTe single quantum wells," Optical and Quantum Electronics, vol. 41, no. 7, pp. 559-565, 2009.

[23] A. Sali, M. Fliyou, H. Satori, and H. Loumrhari, "The effect of a strong magnetic field on the binding energy and the photoionization process in quantum-well wires," Journal of Physics and Chemistry of Solids, vol. 64, no. 1, pp. 31-41, 2003.

[24] Y.-B. Yu, S.-N. Zhu, and K.-X. Guo, "Polaron effects on thirdharmonic generation in cylindrical quantum-well wires," Solid State Communications, vol. 132, no. 10, pp. 689-692, 2004.

[25] Z. Chen, D. Yao, X. Zhang, and T. Fang, "Polaron effectdependent third-order optical susceptibility in a $\mathrm{ZnS} / \mathrm{CdSe}$ quantum dot quantum well," Microelectronics Journal, vol. 39, no. 12, pp. 1654-1658, 2008. 

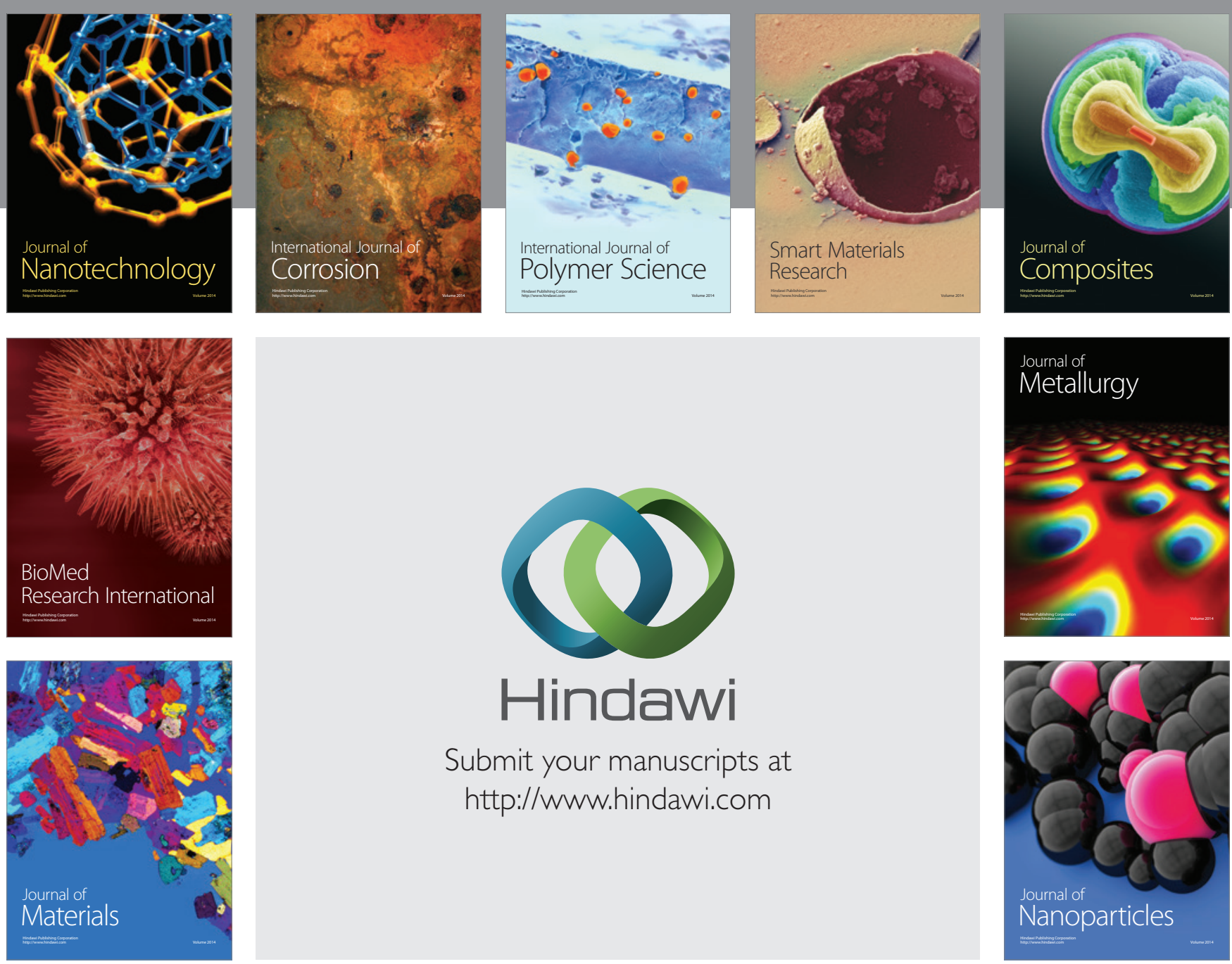

Submit your manuscripts at http://www.hindawi.com
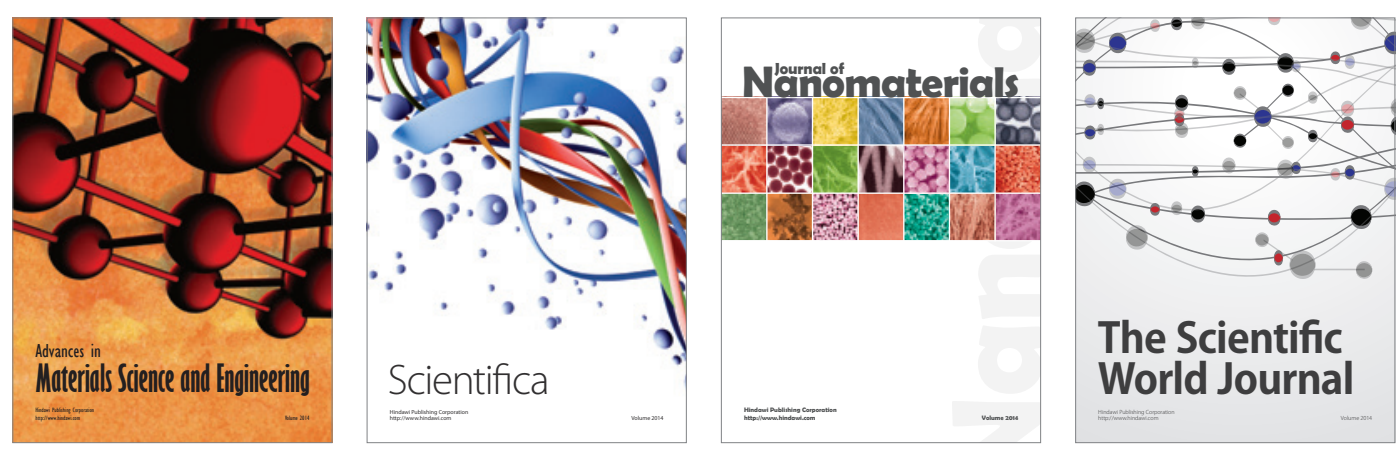

\section{The Scientific World Journal}
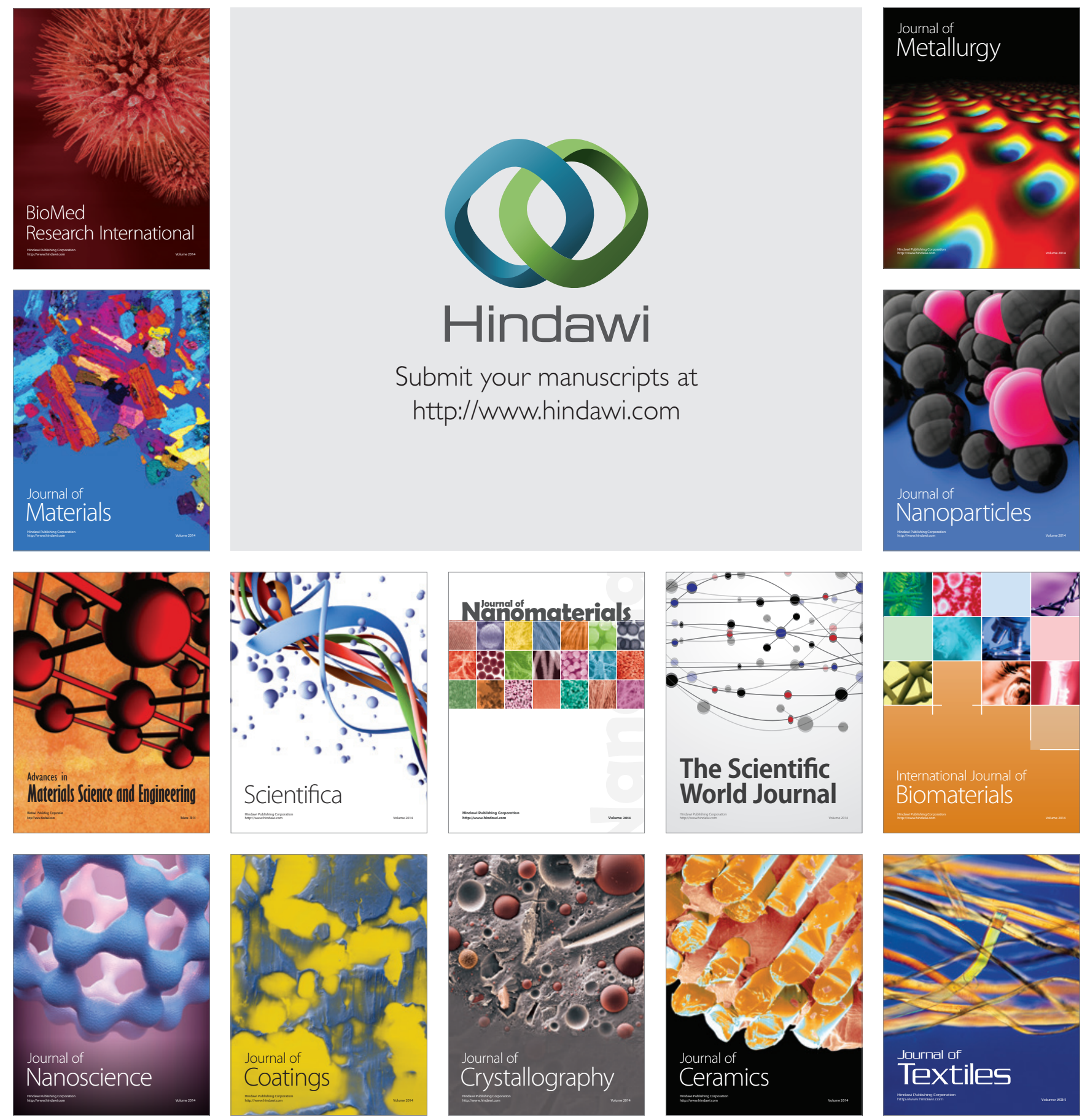